\title{
GINER DE LOS RÍOS, CRÍTICO LITERARIO
}

\author{
JOAQUÍN JUAN PENALVA \\ Universidad Miguel Hernández (Elche)
}

\section{Resumen}

El artículo ofrece un repaso por las principales aportaciones de Francisco Giner de los Ríos en el ámbito de la crítica, la historia y la estética literarias, tarea que desempeñó fundamentalmente en la Revista Meridional de Granada entre 1862 y 1863 y en algunos periódicos de Madrid. Esos trabajos, sumados a otros posteriores, conformaron su primer libro, Estudios literarios (1866), que tuvo una segunda edición ampliada en 1876, con el título de Estudios de literatura y artes. Después de 1876, Giner de los Ríos abandonó los estudios literarios, aunque regresó a ellos poco después, cuando publicó sus «Cartas literarias» en El Pueblo Español. Esta nueva serie de textos apareció a finales de 1878 y principios de 1879, y suponen su última aportación a la crítica literaria. Entre los autores de los que se ocupa, destacan Ventura Ruiz Aguilera, a quien le dedica cuatro artículos, y Benito Pérez Galdós.

Palabras clave: Francisco Giner de los Ríos; crítica literaria; historia literaria; estética literaria.

\begin{abstract}
This paper presents a review of the main contributions that Francisco Giner de los Ríos made in the field of literary criticism, history and aesthetics, which he mainly carried out in the Revista Meridional of Granada between 1862 and 1863, and later in some newspapers from Madrid. All these works and some he wrote afterwards were compiled in Estudios literarios (1866), his first published book, which had an extended edition in 1876, Estudios de literatura y artes. After 1876, Giner de los Ríos left the field of literary studies, but he came back soon, when he published «Cartas literarias» at El Pueblo Español. These new writings appeared at the end of 1878 and beginning of 1879 , and they were Giner's last contribution to the literary criticism. Among the authors he wrote about, we can emphasize Ventura Ruiz Aguilera (Giner wrote four articles about him) and Benito Pérez Galdós.
\end{abstract}

Keywords: Francisco Giner de los Ríos; literary criticism; literary history; literary aesthetics.

Anales, 27 (2015), pp. 131-138

DOI: 10.14198/ALEUA.2015.27.07 
Aunque hoy en día es más recordado como uno de los fundadores de la Institución Libre de Enseñanza, Francisco Giner de los Ríos también ejerció, durante algunas etapas de su vida, de crítico literario. Publicó sus primeros artículos en Granada, en cuya universidad estudiaba desde 1853, concretamente en la Revista Meridional, en la que escribió fundamentalmente sobre política y literatura. Giner nunca formuló un sistema filosófico propio, pero su vida fue ejemplo de cuanto creía y defendía — no en vano los profesores krausistas eran considerados textos vivos-. En sus primeros años en Madrid, lugar al que se trasladó en 1863, conoció a Gumersindo de Azcárate, con quien inició varias empresas. El verdadero maestro de Giner fue Julián Sanz del Río, importador de la filosofía krausista, a quien le unió una estrecha relación entre 1863 y 1869.

Giner de los Ríos fue un autor muy polifacético. Valorado fundamentalmente por su labor como pedagogo y guía de hombres, caló hondo en todos sus discípulos, que lo consideraron, más que un escritor o un filósofo, un conversador y un amigo. El testimonio de quienes lo conocieron, si bien es fundamental, fue siempre encomiástico y admirativo, lo que, en ocasiones, ha impedido una aproximación objetiva a su obra escrita, que según esos mismos seguidores es tan solo un tímido reflejo de las ideas del maestro. No necesita Giner de tal cautela, pues su producción, variada y extensa, se defiende por sí sola. Otra cosa es que no podamos valorar en su justo término su importancia como texto vivo, pues de esa faceta suya solo contamos con las apreciaciones de quienes tanto le quisieron.

Además de pedagogo, Giner fue también filósofo, sociólogo y jurista, pero sus escritos no se circunscribieron únicamente a esas disciplinas, sino que abordaron también la crítica e historia literarias, el arte, la rejería, la tapicería, el mobiliario y las custodias de las iglesias, entre otros muchos y pintorescos temas. Tradujo la obra de algunos autores extranjeros, sobre todo filósofos, entre los que podemos destacar a Krause, Leonhardi, Ahrens, Roeder, Falckenberh y Compayré. A lo largo de toda su vida colaboró en diversas publicaciones periódicas como Revista General de Legislación y Jurisprudencia, El Reino, Revista Meridional, Revista Popular, Ilustración Artística, La Lectura, La España Moderna, El Pueblo Español, Revista Mensual de Filosofía, Literatura y Ciencias de Sevilla, Revista de Educación, Revista de España... Además, contribuyó a la creación de dos nuevas tribunas, en este caso científicas: el Boletín-Revista de la Universidad de Madrid y el Boletín de la Institución Libre de Enseñanza.

La fundación de la Institución Libre de Enseñanza supuso un hito en su trayectoria intelectual, según reconocen todos los estudiosos de la 
figura de Giner. Así, a partir de 1876 sus escritos se orientaron fundamental, aunque no exclusivamente, hacia la pedagogía. Ahora bien, los primeros artículos publicados por Giner versaron sobre crítica, historia y estética literarias, y aparecieron en la Revista Meridional de Granada. Esos trabajos, sumados a algunos posteriores, conformaron el volumen Estudios literarios (1866), a la sazón, su primer libro publicado, que tuvo una segunda edición ampliada en 1876, con el título de Estudios de literatura $y$ artes.

Desde 1868 centró gran parte de sus esfuerzos en estudios de carácter filosófico y jurídico, relacionados con su cátedra de Filosofía del Derecho y Derecho Internacional. De esta época son sus libros Introducción a la filosofía del derecho (1869), Enciclopedia del derecho (1870), Principios elementales del derecho (1871), Lecciones sumarias de psicología (1874) y Estudios filosóficos y religiosos (1876).

En 1880, Giner pronunció el discurso de apertura de curso en la ILE, publicado con el título de El espíritu de la educación en la Institución Libre de Enseñanza. A partir de ese momento, el tema de la pedagogía, que ya había estado presente en algunos de sus libros de la década anterior, cobró una importancia fundamental: La futura ley de Instrucción Pública (1869), Los estudios de facultad (1870), Instrucción y educación (1879), Enseñanza y educación (1881), La enseñanza confesional y la escuela (1882), Campos escolares (1884), Las reformas del señor Pidal en la enseñanza de las maestras (1885), Estudios sobre educación (1886), El espíritu mecánico en la educación (1887) y Educación y enseñanza (1889).

Giner fue colaborador habitual de la Revista de Derecho y Sociología, que comenzó su andadura en 1895. Durante esos años, coincidiendo con el primer lustro de la revista, proliferaron sus trabajos de índole sociológica, reunidos en los volúmenes Las personas sociales en la ética de Wundt (1895), El estado de la persona social (1896), La ciencia como función social (1898) y Estudios y fragmentos sobre la teoría de la persona social (1899). Este interés por la sociología no empañó, sin embargo, su constante preocupación por la pedagogía, asunto central de La enseñanza individual en la escuela (1895), Grados naturales en la educación (1897) y Sobre la enseñanza de la filosofía (1898). De hecho, en la última etapa de su vida Giner se ocupó exclusivamente de esa disciplina en El problema de la educación nacional y las clases "productoras» (1900), Pedagogía correccional (1900), La escuela que «cerrará presidios» (1901) y Pedagogía universitaria (1905).

Los escritos sobre literatura de Giner, pues, se circunscriben al momento de su formación intelectual, cuando más directamente recibe la 
influencia de la filosofía alemana. Reunidos, según se ha dicho, en 1866 (Estudios literarios) y 1876 (Estudios de literatura y artes), casi todos los publicó antes en la Revista Meridional de Granada entre 1862 y 1863, y algunos en periódicos de la corte. El propio autor, en el prólogo al volumen de 1866, indicaba que,

escritos en su mayor parte poco después de los veinte años, edad en que no es dado a la medianía producir sazonados frutos, deben considerarse tan solo como hijos de ese afán que el espíritu siente por representarse sus propias ideas e impresiones, según los acontecimientos de la vida van solicitando su atención y promoviendo en él un cúmulo de reflexiones desordenadas e incompletas (5).

Todos estos trabajos fueron reunidos por Manuel Bartolomé Cossío, su más fiel discípulo, en 1919, en el tomo tercero de las Obras completas de su maestro. Giner consideraba que la literatura de su época se encontraba en crisis, tal como explicó en numerosos textos, entre ellos en el prólogo de 1876, donde hablaba en términos de crisis general de las artes:

El Arte presente es el Arte de la vulgaridad. El drama desciende a una conversación discreta; la ópera, a un espectáculo de física recreativa; la lírica, al álbum de las damas; la pintura, al figurín y al almacén de antigüedades: todo ello, en suma, a fútil pasatiempo, a mero adorno secundario de otras más imperiosas necesidades (XII).

También se ocupó Giner de la historia literaria y de la estética, sobre todo en «El arte y las artes» (1871) y «¿Qué es lo cómico?» (1872). En estética, Giner sigue a Krause y considera que la belleza se identifica con la idea de verdad, bien y Dios. Como resulta habitual en muchos pensadores de formación alemana, las ideas ginerianas destilan cierta galofobia.

Uno de sus artículos más interesantes es «Del género de poesía más propio de nuestro siglo», donde se refiere a la crisis de la literatura, de la que tan solo se escapa la lírica, pues el poeta, «reconcentrado en la expresión artística de los sentimientos que encuentra en su alma, apenas nos ilustra indirectamente acerca de la sociedad a que corresponde, y es un guía menos precioso que los de aquellos otros géneros para el estudio de los tiempos pasados (1876: 63).

Así, aunque considera que la epopeya es la más perfecta de las manifestaciones de la épica —en «De la poesía épica, y en particular, de la epopeya» (1864)—, ya que «[n]o se limita a una esfera particular del mundo que tiene ante sus ojos, sino a todas» (1876: 72), reconoce que a finales del siglo XIX no se dan las condiciones necesarias para su aparición. En 
«Poesía erudita y poesía vulgar» (1863), Giner entra de lleno en la dicotomía clásicos-románticos, tema fundamental de un trabajo más extenso de ese mismo año: «Dos reacciones literarias (clásicos y románticos)». En ambos se cargan las tintas sobre los aspectos negativos del neoclasicismo francés, que abominó de la propia tradición medieval y optó por modelos ajenos.

El artículo más interesante del volumen es «Consideraciones sobre el desarrollo de la literatura moderna» (1862), donde se presenta un complejo panorama de la evolución de la literatura a partir de la Edad Media. Se trata de una literatura lastrada fundamentalmente por el exceso de sentimentalismo, realismo e individualismo, «extravíos» que se propagaron fundamentalmente en Francia. Según Giner, uno de los casos más nocivos de la influencia de la literatura francesa sobre la española fue la del romanticismo español, que «no expresa el progreso de nuestros antecedentes nacionales; sino, con notables excepciones, la continuación, naturalmente algo degenerada, del neo-romanticismo francés» (1876: 230). Para Giner, toda creación artística que aspire a permanecer en la historia «debe por un lado, referirse a las leyes necesarias de lo bello; por otro, al carácter de la civilización en que nace: lo inmutable y lo temporal, lo accidental y lo absoluto han de tener en ella representación» (1876: 241).

Junto a estos trabajos de índole histórica y teórica, también se incluyó en este volumen una serie de reseñas. La primera, «Una dolora en prosa» (1865), es un comentario de Lo absoluto, de Ramón de Campoamor. Elogia Giner el propósito divulgativo del libro, que se sitúa dentro de la corriente idealista, y lo califica de poema didáctico, «aquel noble género que aspira a representar sensiblemente la belleza de las ideas y los sentimientos que su contemplación interior despierta en el espíritu del hombre» (1876: 259).

Hasta cuatro artículos llegó a dedicarle Giner de los Ríos, entre 1862 y 1867, a los libros de poemas de su amigo Ventura Ruiz Aguilera. Se detiene especialmente en Elegías, donde Ruiz Aguilera pretendió atrapar la memoria de la hija muerta, y no tanto en Armonías y Cantares, Proverbios ejemplares y La Arcadia moderna: Églogas e Idilios realistas y Epigramas. En todos ellos, Giner elogia desmesuradamente la obra de su amigo, hasta el extremo de afirmar que su poesía debería recitarse en las plazas públicas.

Aparecen también en este volumen dos notas dedicadas a sendos estudios sobre el Quijote: La Estafeta de Urganda o Aviso de Cid Asam-Ouzad Benengeli sobre el desencanto del Quijote, de Nicolás Díaz de Benjumea, y El Quijote y la Estafeta de Urganda, de Francisco María Tubino. En ambos casos, Giner subraya la multiplicidad de lecturas de la novela de Cervantes, 
que trasciende la crítica o parodia de los libros de caballerías. Díaz de Benjumea, en palabras de Giner, aboga por la «identidad entre el mismo Cervantes y su héroe, considerando las aventuras de este como el trasunto alegórico de la infortunada vida del príncipe de nuestros ingenios» (1876: 301), mientras que Tubino cree que «la novela de Cervantes no es directamente sino una crítica literaria, por más que, en forma mediata, contenga otros sentidos diversos, germinación espontánea del genio de su autor» (1876: 307).

La última reseña incluida en Estudios de literatura y artes es la titulada «Un novelista español» (1871), que se centra en La Fontana de Oro, la primera novela de Benito Pérez Galdós. Giner la considera interesante porque se refiere «a un período tan memorable en nuestra historia contemporánea como el del 20 al 23, ofreciendo un animado cuadro de la vida y cultura que por entonces ofrecía esa postrada sociedad, a trechos galvanizada por los sacudimientos políticos» (1876: 313). Alaba sobre todo la creación del personaje de Clara y el sello castizo de que está impregnada toda la obra.

Después de 1876, Giner de los Ríos abandonó los estudios literarios, a los que regresó poco después, cuando publicó sus «Cartas literarias» en El Pueblo Español. Esta nueva serie de textos apareció a finales de 1878 y principios de 1879, y suponen su última aportación a la crítica literaria. Esas «cartas» fueron recogidas, junto a los Estudios sobre artes industriales (1892), en el volumen XV de sus Obras completas, publicado en 1926, y se pueden dividir en dos grupos. El primero lo conforman «Sobre el discurso de apertura de la Universidad», «Sobre la Institución y el Conservatorio», «Sobre publicaciones de Historia», «El Ateneo de Madrid y el Sr. Moreno Nieto», «Sobre la Sociedad de Bibliófilos» y «La "Biblioteca de Autores Aragoneses"». Los títulos de los artículos son bastante descriptivos y no hace falta entrar en más detalles. El segundo grupo incluiría los estudios y reseñas propiamente literarios.

El primer trabajo de esta índole es «Poesías de Flores Arenas», donde Giner recorre brevemente la producción lírica y dramática de este autor, a propósito de la aparición de sus Obras escogidas, publicadas por la Real Academia Gaditana de Ciencias y Letras en edición no venal. En «Sobre La familia de León Roch» vuelve a la novelística de Pérez Galdós y subraya la superioridad de los personajes femeninos sobre los masculinos, una idea que ha asumido unánimemente la crítica posterior. La considera una novela de tesis, pues «está toda ella subordinada a un fin moral: mostrar cómo en España la religión, el principio mismo del amor y concordia entre los hombres, se convierte hoy en potencia diabólica de perversidad y de 
odio» (1926: 287). Además, ataca la caracterización del personaje principal, así como la sensación de "galería de retratos» (1926: 289) que se desprende de toda la obra. Más general es su trabajo «Sobre publicaciones extranjeras», un breve pero completo recorrido por las novedades editoriales que habían ido apareciendo en toda Europa a lo largo de 1878. En «Sobre el teatro» se ocupa de las causas que han motivado la crisis del género dramático en España, agravada por la ausencia de grandes intérpretes, capaces de dignificar con su trabajo producciones mediocres, y por la práctica inexistencia de la figura del director de teatro, encargado de elegir las obras de acuerdo con un programa que atienda a criterios estéticos y ofrezca suficiente variedad en sus contenidos. La última de las «cartas literarias» publicadas en El Pueblo Español se titula «Sobre el libro de González Serrano Goethe», donde Giner, con ocasión del lanzamiento de dicho volumen, aprovecha para regresar a uno de sus autores alemanes predilectos.

Tras este paréntesis, Giner volvió a sus quehaceres habituales y redujo ya definitivamente sus opiniones literarias al ámbito privado de conversaciones y correspondencia. Así, en carta a Clarín de 18 de agosto de 1891, confesaba no estar al día en cuanto a novedades editoriales. Había leído Su único hijo y le elogiaba a su autor la «nota amarga, pesimista, humana» (1965: 113) de la novela. No obstante, Giner consideraba que el género se encontraba en crisis, a la que había contribuido en buena medida la tendencia naturalista: «Nunca he visto como hoy tanta y tan elevada idealidad, y al par tanto rebuscamiento, mezclado con ella, de fotografiar el lado desagradable, animal y grosero de la vida» (1965: 113). Entre la correspondencia de Giner se conservan también dos breves notas dirigidas a Azorín, que le había enviado sendos ejemplares de España y de Lecturas Españolas (1965: 122-123).

Sin duda, el mejor trabajo sobre la crítica literaria gineriana es «Las ideas literarias de Francisco Giner de los Ríos», de Juan López-Morillas. Allí se afirma que el ideal de arte de Giner pasa por la «entrañada conexión entre lo esencial y lo formal» (López-Morillas, 1966: 36). El arte sería, por tanto, «la suprema actividad humana» (1966: 37) y la literatura un elemento fundamental para reconstruir la historia íntima o interna de los pueblos. Pero eso no actúa en detrimento del valor estético propio de la obra literaria, que ha de aunar el fin estético con el fin moral. Según López-Morillas, Giner aspiraba a tres fines: «a) mostrar que la cultura francesa es nociva para la española; $b$ ) alentar el estudio crítico del pasado literario español como inmejorable remedio contra la manía de imitar; y c) 
instar al literato a ser fiel a su espíritu y a su tiempo» (1966: 48). Estos tres objetivos tenían como meta la resolución de la crisis de las letras españolas de acuerdo con el carácter optimista que caracterizaba al pensamiento krausista. Para Giner, la crítica literaria se encontraba en estrecha relación con la filosofía, y el fin de ambas tendría carácter ético.

Más recientemente, Adolfo Sotelo Vázquez se ha ocupado del mismo tema en el artículo «Las ideas literarias de don Francisco Giner de los Ríos» (2002), si bien se centra fundamentalmente en las opiniones de Francisco Giner de los Ríos sobre la novelística de Galdós y su relación con las de otros críticos de la época.

Lo cierto es que, desde su perspectiva idealista, Giner diagnosticó muchos de los males de la literatura moderna y analizó con solvencia unas cuantas obras, si bien, como apuntaba ya López-Morillas en su momento, «el interés de Giner por la literatura, no obstante ser profundo, queda siempre subordinado a su interés por la filosofía, entendida esta en el doble sentido de hermenéutica histórica y de propedéutica de una vida humana plena y armónica» (1966: 55).

\section{Bibliografía citada}

GINER DE LOS Ríos, F, Estudios literarios, Madrid, Imprenta de R. Labajos, 1866.

- Estudios de literatura y artes, Madrid, J. M. Pérez, 1876.

- Obras completas de D. Francisco Giner de los Ríos. XV. Estudios sobre artes industriales y Cartas literarias, Madrid, La Lectura, 1926.

- Ensayos y cartas: Edición de homenaje en el cincuentenario de su muerte, México, Fondo de Cultura Económica, 1965.

- Ensayos, ed. Juan López-Morillas, Madrid, Alianza, 1969.

LÓPEZ-Morillas, J., «Las ideas literarias de Francisco Giner de los Ríos», Revista de Occidente, año IV, 2. ${ }^{a}$ época, 34 (1966), pp. 32-57.

Sotelo VÁzQuez, A., «Las ideas literarias de don Francisco Giner de los Ríos», en La elaboración del canon en la literatura española del siglo XIX, Barcelona, Universitat de Barcelona, 2002, pp. 407-414.

Fecha de recepción: 17-11-2015

Fecha de aceptación: 24-11-2015 